\title{
73. SOBRE LA FLORA DE LA PROVINCIA DE LEÓN
}

\author{
Raquel ALONSO REDONDO, Elena de PAZ CANURIA, \\ Emilio PUENTE GARCÍA y Angel PENAS MERINO
}

About the flora of the León province.

Palabras clave. Corología, León, España, Flora iberica.

Key words. Chorology, León, Spain, Flora iberica.

Consultados los tomos de Flora iberica, Castroviejo et al.(1986-1997), publicados hasta la fecha (I, II, III, IV, V y VIII), se han observado ciertas imprecisiones en las áreas de distribución de algunos táxones para la provincia de León.

Para subsanar en la medida de lo posible estas imprecisiones, se ha procedido a la revisión correspondiente del material del Herbario LEB. Como resultado de la misma se ha elaborado una relación de táxones de los cuales tenemos constancia fiable de su presencia en la provincia a través de dicho material.

Señalamos con un asterisco $(*)$ los táxones para los que Flora iberica no indica la sigla provincial Le. En este caso referenciamos las citas bibliográficas conocidas. Aquéllos señalados para nuestra provincia con dudas geográficas o taxonómicas -Le?- aparecen precedidos por el símbolo (+). La relación se completa con los táxones para los que Flora iberica basa su presencia en esta provincia en el testimonio de un especialista o en alguna cita fiable, pero no respaldada por pliegos (Le)-.

En todos los casos se especifican los datos que constan en las etiquetas de los pliegos de herbario, a saber: localidad, coordenadas UTM, fecha de recolección, ecología, altitud, leg. y número de LEB.

\section{* Adonis annua L.}

LEÓN: Monte Grande. Valencia de Don Juan
(30TTM9479); 4-VI-1995. Sobre margas con cantos, arado. E. de Paz. LEB 59460.

\section{* Althaea hirsuta L.}

LEÓN: Carbajal de Fuentes. 23-V-1974. J. Andrés. LEB 10601. Cistierna (30TUN24); 30-V1987. Pastizales sobre sustrato calizo. E. Puente. LEB 39265. Cistierna; 12-VI-1969. Mayor, Andrés \& Carbó. LEB 2192; La Robla (30TTN84); 26-VI1983. Encinar. C. Pérez Morales. LEB 24931. Lancia (30TUN003112); 22-V-1993. Pastizal. E. de Paz. LEB 55994. Los Corrales del Hoyo. El Valle de las Casas (30TUN3138); 15-VI-1996. Cultivo abandonado. $1105 \mathrm{~m}$. R. Alonso. LEB 61630. Mirantes de Luna; VI-1973. Romero. LEB 2194. Mirantes de Luna; VII-1973. Romero. LEB 2195. Monte del Duque. Valderas (30TTM956667); 22 V-1996. Pastizal de encinar. E. de Paz. LEB 59587. Monte Grande. Valencia de Don Juan (30TTM9479); 4-VI-1995. Sobre margas con cantos, arado. E. de Paz. LEB 59467. Monte Grande. Valencia de Don Juan; 25-V-1977. Borde de encinar. A. Penas. LEB 6324. Pola de Gordón (30TTN84); 23-V-1987. Pastizales sobre sustrato calizo. E. Puente et al. LEB 45217. Santa Lucía de Gordón (30TTN85); 13-VI-1977. Encinar. C. Pérez Morales. LEB 24987. Valmartino (30TUN2740); 15-VI-1996. Tomillar. 1100 m. R. Alonso. LEB 63441 . Valle de la Duerna. Santa Olaja de la Varga (30TUN2847); 16-VI-1997. Pastizal entre calizas. $1240 \mathrm{~m}$. R. Alonso. LEB 63459. Villacontilde (30TUN01); 7-VI-1988. A. Penas et al. LEB 34778.

Citas bibliográficas: Pérez Morales (1988). Aedo et al. (1994) ya señalan que falta la sigla provincial.

* Althaea officinalis L.

LEÓN: Ardoncino (30TTN80); 1-VIII-1982. 
Pradera juncal. R. García Cachán. LEB 13112. Gordoncillo (30TUM06); 21-IX-1986. MolinioArrhenatheretea. 750 m. M.E. García. LEB 36427. Jabares de los Oteros (30TTM99); 24-VII-1985. Cirsio-Holoschoenetum. 775 m. M.E. García. LEB 40399. Jabares de los Oteros (30TTM99); 24-VII1985. Cirsio-Holoschoenetum. M.E. García. LEB 49429. Jabares de los Oteros (30TTM99); 24-VII1985. Cirsio-Holoschoenetum. M.E. García. LEB 49479. Jabares de los Oteros (30TTM99); 24-VII1985. Cirsio-Holoschoenetum. M.E. García. LEB 49823. Santibáñez de Porma; 14-VI-1970. J. Andrés \& R. Carbó. LEB 2196. Valencia de Don Juan; $27-$ VII-1976. A. Penas. LEB 6325. Valjunco. Valencia de Don Juan (30TTM98); 27-VII-1976. A. Penas. LEB 41709. Valjunco. Valencia de Don Juan; 27 VII-1976. A. Penas. LEB 2197.

Citas bibliográficas: Penas Merino (1984).

Alliaria petiolata (Bieb.) Cavara \& Grande LEÓN: Cistierna (30TUN2640); 12-IV-1997. Ruderal. 940 m. R. Alonso. LEB 6346. Chana de Somoza; 1-V-1975. Borde de pared. F. Llamas. LEB 7454. Devesa de Curueño; 11-V-1969. Encinar. J. Andrés \& R. Carbó. LEB 1019. Geras (30TTN75); 12-VII-1977. Borde de camino. C. Pérez Morales. LEB 25789. La Martina (29TPG91); 11-V-1986. Alliarion. 500 m. M.E. García. LEB 33084. Lago de Carucedo (29TQH80); 18-IX-1983. Pinar. M.E. García. LEB 20110. Las Omañas; 12-V-1973. A. Pérez. LEB 1022. Mansilla de las Mulas; 2-V-1971. J. Andrés. LEB 10568. Olleros-Riaño. Km. 1; 5-VI1983. T.E. Díaz, A. Penas \& M.E. García. LEB 27125. Palacios del Sil (29TQH05); 28-V-1981. Herbazales escionitrófilos. E. Puente. LEB 12455. Pico del Aguila. Llombera (30TTN8946); 5-V-1992. Melojar. 1250 m. G. González Sierra. LEB 54020. Puebla de Lillo; 15-VII-1975. E. Hernández. LEB 10439. Rioscuro-Villaseca de Laciana (29TQH25); 16-IV-1983. Herbazales escionitrófilos. E. Puente. LEB 15737. San Clemente de Valdueza (29TQH0205); 16-IV-1991.712 m. F. Llamas \& C. Acedo. LEB 49075. San Martín de la Falamosa; 12V-1973. A. Pérez. LEB 1023. Torneros; 25-V-1977. Borde de carretera. A. Penas. LEB 5980. Torneros; 25-VII-1977. Borde de carretera. A. Penas. LEB 8948. Valdelugueros (30TUN06); 17-V-1978. Seto. M.J. López Pacheco. LEB 16747. Vegas del Condado; 15-V-1969. J. Andrés \& R. Carbó. LEB 1020. Vegas del Condado; 5-VII-1968. J. Andrés.
LEB 22948. Verdiago (30TUN2548); 8-VI-1995. Ruderal. 980 m. R. Alonso. LEB 63448. Viaducto de Aralla; 1-VII-1971. J. Andrés \& R. Carbó. LEB 1021. Villamoros de las Regueras (30TTN92); 21 IV-1994. Borde de chopera. E. de Paz. LEB 59794.

\section{Androsace lactea $\mathrm{L}$.}

LEÓN: Pico Huevo (30TTN96); 1-VIII-1979. Pastizales sobre sustratos calizos. 1900 m. M.J. López Pacheco. LEB 11291.

\section{+ Anemone palmata $\mathrm{L}$}

LEÓN: Castrillo de Cepeda (29TQH41); 16V-1985. Laguna. A. Penas, M.E. García, L. Herrero, M. Garzón \& I. Jiménez. LEB 15978. El Chopo. Villasabariego (30TUN028131); 8-IV-1993. Encinar. E. de Paz. LEB 56025. Herreros de Jamuz (30TTM58); 14-V-1986. Encinar. 790 m. M.E. García. LEB 32978. Herreros de Jamuz; 6-V-1973. J. Andrés \& R. Carbó. LEB 570. Herreros de Jamuz; 6-V-1973. J. Andrés. LEB 10228. La Nora (30TTM67); 14-V-1987. Encinar-jaral. A. Penas et al. LEB 41207. Montejos del Camino (30TTN81); 30-IV-1985. Brezal. A. Penas, M.E. García \& M. Garzón. LEB 15979. Montejos del Camino; 10-IV 1987. Claro de matorral sobre suelo ácido. F. Gómiz. LEB 35543. Santiago Millas; 11-V-1977. F. Llamas. LEB 7478. Santiago Millas-Curillas (29TQG3995); 23-VI-1994. Sobre terreno silíceo en pradera. 920 m. F. Gómiz. LEB 59224. Villadangos del Páramo (30TTN71); 30-IV-1985. Encinar. A. Penas, M.E. García \& M. Garzón. LEB 29488.

\section{Anogramma leptophylla (L.) Link}

LEÓN: Cuevas del Sil (29TQH15); 28-V-1981. Fisura de roca silícea. E. Puente. LEB 12578. Pantano de Bárcena; 17-IV-1983. Cuarcitas. T.E. Díaz et al. LEB 15210. Pombriego (29TPG89); 12 VII-1985. Olmeda. I. Jiménez. LEB 32487. Tejedo del Sil (29TQH15); 2-VII-1983. Talud terroso ombrófilo silíceo. E. Puente. LEB 16833. Yebra (29TPG99); 7-V-1985. Talud terroso. I. Jiménez. LEB 32485.

Arenaria leptoclados (Reichenb.) Guss.

LEÓN: La Llama-Prado de la Guzpeña (30TUN3438); 25-V-1996. Pastizal terofítico en claro de encinar muy abierto. $1095 \mathrm{~m}$. R. Alonso. LEB 62445. Lancia (30TUN003112); 22-V-1993. Pastizal de diente. E. de Paz. LEB 55996. Lancia 
(30TUN003112); 29-IV-1994. Pastizal de diente. E. de Paz. LEB 59786. San Miguel de Escalada (30TUN111158); 3-VI-1996. Pastizal. 860 m. E. de Paz. LEB 59691. Villafer (30TTM902656); 29-V1994. Tomillar. $790 \mathrm{~m}$. E. de Paz. LEB 59381.Villagallegos (30TTM89); 4-VI-1995. Pastizal. E. de Paz. LEB 59448.

* Arenaria obtusiflora G. Kunze subsp. ciliaris (Loscos) Font Quer

LEÓN: Cistierna; 30-V-1982. F. Llamas. LEB 15538. Cistierna; 30-V-1982. F. Llamas. LEB 7727. Riaño; 12-VI-1969. J. Andrés \& R. Carbó. LEB 18112. Riaño; 12-VI-1969. J. Andrés \& R. Carbó. LEB 18160. Valle del Molino. Valmartino-El Valle de las Casas (30TUN2838); 17-VI-1997. Pastizal anual en zona arenosa con humedad temporal. 1050 m. R. Alonso. LEB 63476.

Citas bibliográficas: Llamas et al. (1984).

Athyrium distentifolium Tausch ex Opiz

LEÓN: Valle de Lechada. Macizo de Peña Prieta. Boca de Huérgano (30TUN5764); 14-VII1990. Entre grandes bloques de granitos. $2100 \mathrm{~m}$. M.L. Gil Zúñiga \& J.A. Alejandre. LEB 47354.

* Atriplex prostrata Boucher ex DC.

LEÓN: Bercianos del Real Camino (30TUM24); 5-VI-1981. Borde de laguna y zona de transición. M. Fernández Aláez. LEB 36196. Laguna Grande. Bercianos del Real Camino (30TUM29); 2VI-1983. T.E. Diaz et al. LEB 45246.

\section{* Atriplex rosea $\mathrm{L}$.}

LEÓN: Polígono de Renueva. León. 13-IX1973. F. Llamas. LEB 12050.

\section{* Biscutella auriculata L.}

LEÓN: Carbajal de Fuentes (30TTM97); 27 IV-1990. F. Llamas \& C. Acedo. LEB 55118. El Portillo (30TTN81); 24-V-1986. RuderaliSecalietea. 840 m. M.E. García. LEB 33014. El Portillo (30TTN91); 24-V-1986. M.E. García. LEB 50284. Fuentes de Carbajal; 18-V-1974. E. Hernández. LEB 37941. Matallana de Valmadrigal (30TUM09); 2-VI-1983. T.E. Díaz, A. Penas \& M.E. García. LEB 42055. Matallana de Valmadrigal; 2-VI-1983. T.E. Díaz, A. Penas, E. Puente \& M.E. García. LEB 26850. Monte Grande. Valencia de Don Juan; 3-VI-1976. A. Penas. LEB 1066. Puente
Villarente (30TTN91); 4-VI-1986. J. Andrés. LEB 43948. Puente Villarente (30TTN91); 4-VI-1986. Ruderal. M.E. García. LEB 49816. Quintanilla de los Oteros (30TTM995868); 12-V-1993. Cárcava calcárea. E. de Paz. LEB 56010. Valderas (30TTM96); 10-VI-1979. J. Andrés. LEB 42109. Villaquejida (30TTM833705); 10-VI-1993. Barbecho. E. de Paz. LEB 55960.

Citas bibliográficas: Carbó Nadal et al. (1977). Pérez Morales (1988).

\section{* Brassica barrelieri (L.) Janka}

LEÓN: Destriana (29TQG39); 11-V-1977. F Llamas. LEB 60281. Destriana; 11-V-1977. F. Llamas. LEB 7473. Toralino de la Vega (30TTM49); 14-V-1972. J. Andrés. LEB 48802. Toralino de la Vega; 14-V-1972. E. Hernández. LEB 37951. Toralino de la Vega; 14-V-1972. E. Hernández. LEB 37952.

Citas bibliográficas: Llamas (1984: 70). Herrero et al. (1988: 320).

\section{Camphorosma monspeliaca $\mathrm{L}$.}

LEÓN: Valdefuentes (30TUM06); 4-IX-1985.

A. Penas et al. LEB 29420.

\section{Ceratophyllum demersum $\mathrm{L}$.}

LEÓN: Villadangos (30TTN721114); 30-VIII1981. Interior de la laguna. M. Fernández Aláez. LEB 45380. Zotes del Páramo (30TTM744841); 16 VII-1983. Interior de la laguna. Fernández Aláez. LEB 48769.

\section{* Cistus crispus L.}

LEÓN: Estébanez de la Calzada (30TTN50); 6-VI-1987. 860 m. A. Penas et al. LEB 34885.

Citas bibliográficas: Pérez Morales et al. (1993).

\section{Consolida pubescens (DC.) Soó}

LEÓN: Vallecillo (30TTM19); 24-VI-1986. Borde de camino. 850 m. M.E. García. LEB 35756.

* Coronopus didymus (L.) Sm.

LEÓN: Busdongo (30TTN76); 5-X-1983. Medios pisoteados. C. Pérez Morales. LEB 25905. La Martina (29TPH91); 15-V-1988. Ruderal. A. Penas et al. LEB 33707. Matallana de Torío; 29VIII-1979. F. Llamas. LEB 25535.

Citas bibliográficas: Pérez Morales (1988). Aedo et al. (1994) ya indican la omisión de la sigla provincial. 
* Coronopus squamatus (Forsskal) Ascherson

LEÓN: Castrovega de Valmadrigal (30TUM18); 7-VII-1985. A. Penas, M.E. García \& L. Herrero. LEB 29507. Cospedal de Babia (29TQH4062); 27-VIII-1989. Comunidades de suelos compactados por el pisoteo. E. Puente \& $M^{a} J$. López Pacheco. LEB 63477. Lodares; 4-IX1978. E. Hernández. LEB 317. Riaño (30TUN3660); 5-VIII-1996. Ruderal. 1140 m. R. Alonso. LEB 63468. Vegas del Condado; 22-V-1969. J. Andrés \& R. Carbó. LEB 1114.

Citas bibliográficas: Carbó et al. (1972). Penas Merino et al. (1987-88). Aedo et al. (1997) ya indican la omisión de la sigla provincial.

Chaetonychia cymosa (L.) Sweet

LEÓN: Marrablanca. Felechares de la Valdería (29TQG47); 23-VI-1987. Isoeto. 860 m. A. Penas, M.E. García \& L. Herrero. LEB 34745.

* Chamaesyce canescens (L.) Prokh. subsp. canescens

LEÓN: La Bañeza (30TTM68); 27-VIII-1995. Cultivos abandonados. C. Acedo. LEB 55049.

\section{* Chenopodium hybridum $\mathrm{L}$}

LEÓN: Pío. Oseja de Sajambre; 15-IX-1979.

Nitrófilo. E. Hernández. LEB 21812.

Citas bibliográficas: Hernández Hernández (1985).

\section{* Chenopodium multifidum L.}

LEÓN: Palanquinos (30TTN90); 11-IX-1985. Chenopodion muralis. M.E. García. LEB 31166.

Citas bibliográficas: García González et al. (1987).

\section{* Chenopodium polyspermum L.}

LEÓN: Boca de Huérgano-Puerto del Pando, cruce (30TUN4355); 14-VIII-1996. Ruderal. 1130 m. R. Alonso. LEB 63466. Igüeña (29TQH23); 9 IX-1981. Prado. E. Postigo. LEB 43338. Vega de Infanzones; 24-VIII-1977. A. Penas. LEB 5875.

Citas bibliográficas: Tüxen \& Oberdorfer (1958). Díaz González \& Penas Merino (1984). Pérez Morales (1988). Aedo et al. (1994) ya señalan que falta la sigla provincial.

\section{* Chenopodium vulvaria $\mathrm{L}$.}

LEÓN: El Castillo. Vegarienza (29TPH44); 17-VII-1973. A. Pérez. LEB 46827. El Castillo. Vegarienza (29TPH44); 17-VII-1973. A. Pérez. LEB
8820. Valencia de Don Juan (30TTM98); 23-VIII1978. Como mala hierba de cultivos hortícolas. A. Penas. LEB 4849.

\section{Descurainia sophia (L.) Webb ex Prantl}

LEÓN: Benamariel (30TTM89); 3-V-1986. Ruderali-Secalietea. 750 m. M.E. García. LEB 37005. Monte del Duque. Valencia de Don Juan (30TTM978632); 24-IV-1993. Encinar. E. de Paz. LEB 55783. Valderas; 23-IV-1983. Lugares ruderalizados. T.E. Díaz et al. LEB 15238.

* Diplotaxis erucoides (L.) DC. subsp. erucoides LEÓN: Eras de Renueva. León (30TTN81). 3V-1998. Escombrera. E. Puente. LEB 61546.

* Dryopteris carthusiana (Vill.) H.P. Fuchs LEÓN: Torneros de la Valdería (29TQG27); 4-VII-1986. Canales bajo alisos. 900 m. J. Pérez Carro \& M.P. Fernández Areces. LEB 35465. Citas bibliográficas: Carbó Nadal et al. (1977).

Epilobium tetragonum L. subsp. tetragonum LEÓN: Embalse del Porma; VII-1978. E. Hernández. LEB 18145. Lago de Carucedo (29TPH80); 26-VI-1983. Borde de cañaveral. M.J. López Pacheco. LEB 18627. Laguna Sardón. San Pedro Bercianos (30TTM79); 5-IX-1981. Interior de la laguna desecada. 823 m. Fernández Aláez. LEB 28288. Riaño (30TUN3660); 5-VIII-1996. Ruderal, entre piedras. 1140 m. R. Alonso. LEB 63467.Villadangos; 25-VII-1983. Zonas húmedas. J. Pérez Carro \& P. Fernández Areces. LEB 29176.

\section{* Eruca vesicaria (L.) Cav.}

LEÓN: Besande (30TUN4651); 9-VI-1995. Borde de carretera. $1.300 \mathrm{~m}$. R. Alonso. LEB 63449. Castillo de Valencia de Don Juan; 3-VI-1976. A. Penas. LEB 1139. El Portillo; VII-1977. A. Penas. LEB 5989. Marne (30TTN91); 19-IV-1969. J. Andrés. LEB 60288. Valencia de Don Juan (30TTM9285); 23-IV-1994. Cárcava calcárea. E. de Paz. LEB 59748. Valencia de Don Juan; 16-III1985. En borde de precipicio sobre el río. F. Gómiz. LEB 29069.

Citas bibliográficas: Díaz González \& Penas (1984).

Erysimum cheiri (L.) Crantz

LEÓN: León (30TTN81). 25-V-1986. 
Parietarietea judaicae. 840 m. M.E. García. LEB 35781. LEB 33012.

\section{Euphorbia sulcata De Lens}

LEÓN: Monte del Duque. Valderas (30TTM960660); 24-IV-1993. Encinar. E. de Paz. LEB 56059. Valle del Diez. Valmartino (30TUN2940); 12-IV-1997. Pedregal calizo. 1200 m. R. Alonso. LEB 63463.

\section{Fallopia dumetorum (L.) J. Holub}

LEÓN: Villager de Laciana (29TQH15); 2IX-1981. Cultivado como ornamental. E. Puente. LEB 12544.

\section{* Fumaria capreolata $\mathrm{L}$.}

LEÓN: Embalse de Peñarrubia (29TPH70); 13-V-1987. Ruderali-Secalietea. 400 m. A. Penas et al. LEB 33981. Embalse de Peñarrubia (29TPH70); 21-V-1988. Al pie de cantil calizo, sobre suelo nitrificado. F. Gómiz. LEB 51412.

\section{Halimium ocymoides (Lam.) Willk.}

LEÓN: Jiménez de Jamuz (30TTM58); 19-VI1983. Pinar. T.E. Díaz, A. Penas \& M.E. García. LEB 41409. LEB 49384. Truchillas (29TQG0377); 12-VI-1993. Brezal con orientación sur. 1500 m. A. Penas \& M. de Godos. LEB 56285.

\section{Helianthemum aegyptiacum (L.) Miller}

LEÓN: Benamariel (30TTM89); 9-V-1998. Pastizal terofítico. R. Alonso, E. Alonso \& E. Puente. LEB 61545. Jabares de los Oteros (30TTM935999); 30-V-1993. Pastizal terofítico. E. de Paz. LEB 56411. Maudes. Calzada del Coto (30TUM269917); 31-V-1996. Pastizal terofítico. 815 m. E. de Paz. LEB 59521. Monte de Valderas (30TTM949641); 29-V-1994. Pastizal en claro de encinar. $750 \mathrm{~m}$. E. de Paz. LEB 58938. Picagallinas. Jabares de los Oteros (30TTN001940); 22-V-1996. Pastizal en claro de encinar. E. de Paz. LEB 59628. Villaquejida (30TTM834705); 10-VI-1993. Tomillar. E. de Paz. LEB 59975.

\section{Helianthemum apenninum (L.) Miller subsp. apenninum}

LEÓN: Carrizo de la Ribera (30TTN695180); 30-IV-1993. Cortado en la carretera. E. de Paz. LEB 63240. Cea-San Pedro de Valderaduey (30TUN364033); 26-V-1994 blo de robledal en borde de cultivo. E. de Paz. LEB 63226. Celada de Cea (30TUn395012); 15-VI-1994. Pastizal. E. de Paz. LEB 63229. Colinas de Lancia (30TUN004114); 22-V-1993. Tomillar. E. de Paz. LEB 63237. El Portillín. Villaobispo de las Regueras (30TTN926213); 25-V-1996. Aulagar. E. de Paz. LEB 63224. El Portillín. Villaobispo de las Regueras (30TTN929217); 30-V-1993. Aulagar. E. de Paz. LEB 63235. El Portillo (30TTN918167); 19-VI1993. Pastizal. E. de Paz. LEB 63234. Matallana de Valmadrigal (30TUM0892); 25-IV-1994. Sustrato margoso. E. de Paz. LEB 63238. Maudes. Calzada del Coto (30TUM269917); 31-V-1996. Comunidades de Stipa. E. de Paz. LEB 63223. Monte Pequeño. Valencia de Don Juan (30TTM974834); 19-VI-1993. Encinar. E. de Paz. LEB 63244. Nava de Ios Oteros (30TTM972969); 22-V-1996. Ladera margosa. E. de Paz. LEB 63222. Quintanilla de los Oteros (30TTM98); 9-V-1998. Pastizal basófilo. R. Alonso, E. Alonso \& E. Puente. LEB 61544. Quintanilla de los Oteros (30TTM995868); 12-V-1993. Cárcavas calcáreas. E. de Paz. LEB 63248. Quintanilla de los Oteros (30TTM995868); 12-V-1993. Terraza con carbonatos. E. de Paz. LEB 63242. Villasabariego (30TUN018127); 29-IV-1994. Pastizal. E. de Paz. LEB 63225. Villimer-Castrillo del Porma (30TUN034185); 12-VI-1994. Tomillar. E. de Paz. LEB 63230.

* Helianthemum cinereum (Cav.) Pers. subsp. rotundifolium (Dunal) Greuter \& Burdet

LEÓN: El Chopo. Villasabariego (30TUN029126); 19-VI-1992. Encinar. E. de Paz. LEB 63205. El Chopo. Villasabariego (30TUN029126); 29-IV-1994. Encinar. E. de Paz. LEB 63195. El Portillo (30TTN918167); 19-VI1993. Campo de cultivo abandonado. E. de Paz. LEB 63197. El Portillo (30TTN918167); 3-VII1993. Tomillar. E. de Paz. LEB 63198. La CandamiaPuente Castro (30TTN9118); 30-IV-1994. Aulagar sobre margas. E. de Paz. LEB 63196. Lancia (30TUN004114); 22-V-1993. Tomillar. E. de Paz. LEB 63194. LEB 63201. Matallana de Valmadrigal (30TUM0892); 25-IV-1994. Sustrato margoso. E. de Paz. LEB 63200. Monte Pequeño. Valencia de Don Juan (30TTM974834); 19-VI-1993. Encinar. E. de Paz. LEB 63199. Quintanilla de los Oteros (30TTM98); 9-V-1998. Pastizal basófilo. R. Alonso, E. Alonso \& E. Puente. LEB 61543. Quintanilla de 
los Oteros (30TTM995868); 12-V-1993. Cárcava calcárea. E. de Paz. LEB 63202. Quintanilla de los Oteros (30TTM995868); 12-V-1993. Terraza con carbonatos. E. de Paz. LEB 63204. VillacontildeValle de Mansilla (30TUN0512); 25-VI-1992. Encinar. E. de Paz. LEB 63192. LEB 63203.

\section{* Helianthemum hirtum (L.) Miller}

LEÓN: Corbillos de la Sobarriba (30TTN91); 17-VI-1990. Colina margosa. F. Llamas, C. Acedo \& G. González Sierra. LEB 55046. El Chopo. Villasabariego (30TUN028131); 19-VI-1992. Encinar. E. de Paz. LEB 44198. El Chopo. Villasabariego (30TUN029126); 19-VI-1992. Encinar. E. de Paz. LEB 44197. El Monte Pequeño. Valencia de Don Juan (30TTM974834); 19-VI- 1993. Encinar. E. de Paz. LEB 63213.El Portillín. Villaobispo de las Regueras (30TTN9221); 16-VI1995. Aulagar. E. de Paz. LEB 63208. El Portillín. Villaobispo de las Regueras (30TTN926213); 25 V-1996. Aulagar. E. de Paz. LEB 63206. El Portillín. Villaobispo de las Regueras (30TTN929217); 30V-1993. Aulagar. E. de Paz. LEB 63217. LEB 63210. LEB 63209. LEB 63221. LEB 63220. El Portillo (30TTN918167); 19-VI-1993. Campo de cultivo abandonado. E. de Paz. LEB 63216. LEB 63215. LEB 63214. Herreros de Jamuz; 3-VI-1973. J. Andrés. LEB 10221. LEB 10205. Lancia (30TUN004114); 22-V-1993. Tomillar. E. de Paz. LEB 63218. Monte de Ardoncino; 3-VI-1973. J. Andrés. LEB 10209. Monte del Duque. Valderas (30TTM9566); 8-VI-1994. Pastizal sobre margas. E. de Paz. LEB 58902. Montejos; 2-V-1972. J. Andrés \& R. Carbó. LEB 28906. Nava de los Caballeros (30TUN156234); 16-VII-1992. Piornaljaral. E. de Paz. LEB 63219. Puente Castro; 28-VI1970. J. Andrés \& R. Carbó. LEB 2356. Sahechores (30TUN219218); 27-VI-1995. Tomillar. E. de Paz. LEB 63211. Valdemorilla (30TUM089767); 19-VI1993. Pastizal. E. de Paz. LEB 63212. Valle de Mansilla (30TUN01); 29-V-1990. Encinar. F. Llamas, C. Acedo \& G. González Sierra. LEB 54801. Valle de Mansilla (30TUN01); 29-V-1990. Encinar. F. Llamas, C. Acedo \& G. González Sierra. LEB 55032. Villacontilde-Valle de Mansilla (30TUN0512); 25-VI-1992. Encinar. E. de Paz. LEB 47556. Villasabariego (30TUN018127); 19-VI1992. Tomillar. E. de Paz. LEB 44199.

Citas bibliográficas: Fernández Areces (1990). Carbó Nadal et al. (1977).

\section{* Helianthemum ledifolium (L.) Miller}

LEÓN: Carrizo de la Ribera (30TTN695180); 30-IV-1994. Cortado en la carretera. E. de Paz. LEB 60047. Cota (30TTN0916); 30-VI-1992. Jaralrobledal. E. de Paz. LEB 49064. El Portillo (30TTN918167); 19-VI-1993. Campo de cultivo abandonado. E. de Paz. LEB 60032. La Campana. Colinas de Cea (30TUN339014); 26-V-1994. Tomillar. E. de Paz. LEB 60162. Lancia (30TUN003112); 22-V-1993. Pastizal. E. de Paz. LEB 60049. Monte del Duque. Valderas (30TTM956667); 22-V-1996. Pastizal en claro de encinar. E. de Paz. LEB 59604. Nava de los Oteros (30TTM972969); 22 V-1996. Ladera margosa. E. de Paz. LEB 59643. Quintanilla de los Oteros (30TTM995868); 12-V-1993. Terraza con carbonatos. E. de Paz. LEB 60048.

\section{Helianthemum salicifolium (L.) Mill.}

LEÓN: Cistierna; 28-V-1983. J. Andrés \& F. Llamas. LEB 18391. Colinas de Cea (30TUN339014); 26-IV-1994. Tomillar. E. de Paz. LEB 60161. El Portillín. Villaobispo de las Regueras (30TTN926213); 25-V-1996. Pastizal. E. de Paz. LEB 59673. Las Vallejas. Peñacorada (30TUN3144); 7-VIII-1997. Pastizal terofítico. 1320 m. R. Alonso. LEB 63474. Lugán (30TUN03); 5-VI-1983. Pastizal. T.E. Díaz et al. LEB 45224 Monte Sorriba. Cistierna (30TUN2640); 31-V-1997. Pastizal basófilo. $970 \mathrm{~m}$. R. Alonso. LEB 63461 Nava de los Oteros (30TTM972969); 22-V-1996. Ladera margosa. E. de Paz. LEB 59644. Pola de Gordón (30TTN8149); 29-V-1978. Calizas. E. Puente. LEB 11967. San Juan de Paluezas (29TPH80); 20-IV-1989. F. Llamas \& C. Acedo. LEB 46643. San Martín de la Falamosa; 18-VI1977. A. Pérez. LEB 9178. Valmartino (30TUN2839); 17-VI-1997. Pastizal basófilo. 1075 m. $R$. Alonso. LEB 63460. Valle de Nuestra Señora. Santa Olaja de la Varga (30TUN2746); 16-VI-1997. Pastizal anual basófilo. $1340 \mathrm{~m}$. R. Alonso. LEB 63457. Villanueva del Arbol (30TTN9326); 30-IV1994. Pastizal sobre margas. E. de Paz. LEB 60160.

Helianthemum sanguineum (Lag.) Lag. ex Dunal LEÓN: Benamariel (30TTM89); 4-V-1985. A. Penas, M.E. García \& M. Garzón. LEB 1597.

\section{Hesperis matronalis $\mathrm{L}$.}

LEÓN: Carretera de Caboalles. León. 14-V1972. J. Andrés. LEB 6152. 


\section{* Hypericum calycinum L.}

LEÓN: Morgovejo (30TUN3945); 11-VII-1995. Borde de prado. 1100 m. R. Alonso. LEB 63443.

\section{Hypericum montanum $\mathrm{L}$.}

LEÓN: Garfín; 7-VII-1988. Melojar. A. Penas et al. LEB 45291. La Espina (30TUN4440); 19 VIII-1997. Melojar. 1140 m. R. Alonso. LEB 63470. Peña Derecha. Villarino del Sil (29TQH15); 24VII-1983. Fresneda. E. Puente. LEB 19101.

\section{* Hypericum tomentosum $\mathrm{L}$.}

LEÓN: La Mata del Moral. Villalquite (30TUN11); 7-VII-1988. Pastizal húmedo. A. Penas et al. LEB 44577.

Citas bibliográficas: Pérez Morales et al. (1993).

\section{Isoetes velatum A. Braun subsp. velatum}

LEÓN: Laguna Gállega. Castrillo de Cepeda (29TQH41); 26-IV-1985. A. Penas et al. LEB 15980. Laguna Grande. Valverde Enrique (30TUM18); 21 IV-1988. A. Penas, M.E. García \& L. Herrero. LEB 46678. Laguna Grande. Valverde Enrique (30TUM18); 24-VIII-1988. A. Penas, M.E. García \& L. Herrero. LEB 46679. Valdepolo (30TUN 19); 11-VII-1981. Interior de la laguna. M. Fernández Aláez. LEB 36208. Villadangos del Páramo (30TTN71); 12-VII-1985. Charcas temporales (Preslio-Eleocharidetum palustris). A. Penas, E. Puente \& M.E. García. LEB 35463. Villaverde La Chiquita (30TUN20); 10-VII-1982. Interior de la laguna. M. Fernández Aláez. LEB 36209.

\section{* Juniperus oxycedrus L. subsp. oxycedrus}

LEÓN: Castrillino; 6-XII-1970. Izco, Andrés \& Carbó. LEB 97. Castrillino (30TTN92); 29-I1991. Encinar. F. Llamas \& C. Acedo. LEB 43966. Castrillino (30TTN947258); 22-V-1993. Prebosque. E. de Paz. LEB 56387. Castrillino (30TTN948258); 29-VI-1994. Prebosque sobre margas. E. de Paz. LEB 59709.

Citas bibliográficas: García González (1988)

\section{Lepidium latifolium L.}

LEÓN: León (capital). 25-VII-1984. Baldío. F. Gómiz. LEB 26418.

\section{Lepidium perfoliatum $\mathrm{L}$.}

LEÓN: Herreros de Jamuz; 6-V-1973. J. Andrés. LEB 10219
* Lycopodiella inundata (L.) J. Holub

LEÓN: Suárbol (29TPH74); 9-VI-1986. S. Rivas-Martínez, T.E. Díaz, F. Prieto, J. Loidi \& A. Penas. LEB 46674. Suárbol (29TPH74); 13-VIII1986. Turberas (Erico tetralicis-Trichophoretum (caespitosi) germanici). Rivas-Martínez, T.E. Diaz, F. Prieto, J. Loidi \& A. Penas. LEB 35462. Suárbol (29TPH74); 13-VIII-1986. Turberas (Erico tetralicis-Trichophoretum (caespitosi) germanici). S. Rivas-Martínez, T.E. Díaz, F. Prieto, J. Loidi \& A. Penas. LEB 31831. Suárbol (29TPH74); 13VIII-1986. Turberas (Erico tetralicisTrichophoretum (caespitosi) germanici). S. RivasMartínez, T.E. Díaz, F. Prieto, J. Loidi \& A. Penas. LEB 31830.

Citas bibliográficas: Silva Pando (1994).

* Lychnis coronaria (L.) Desr.

LEÓN: Portilla de la Reina (30TUN4967); 21 VII-1994. Escombrera. 1270 m. R. Alonso. LEB 63451. Puerto de La Magdalena-Rioscuro; VII- 1976. A. Pérez. LEB 8301.

Citas bibliográficas: Fernández Casas \& al., eds. (1994).

\section{Lythrum thymifolia L.}

LEÓN: Carbajal de Fuentes; 20-VII-1978. Zonas encharcadas y luego desecadas. A. Penas. LEB 6377. Matallana de Valmadrigal; 26-VII-1978. A. Penas. LEB 6379. Morales del Arcediano; 14 VII-1977. Charco seco. F. Llamas. LEB 8632. Puente Villarente; 1-X-1970. A. Andrés \& R. Carbó. LEB 2392. Villadangos (30TTN71); 10-VIII-1988. Borde de laguna. A. Penas \& M.E. García. LEB 45276.

\section{Malva tournefortiana $\mathrm{L}$.}

LEÓN: Aralla (30TTN65); 31-VII-1985. Borde de carretera. 1260 m. M.E. García. LEB 36254. Aralla (30TTN65); 31-VII-1985. Borde de carretera. 1260 m. M.E. García. LEB 49084. Balouta (29TPH75); 30-VIII-1985. Onopordetea acanthii. 1100 m. M.E. García. LEB 37118. Destriana; 22VI-1977. F. Llamas. LEB 15195. Monte del Duque. Valderas; 7-VI-1977. A. Penas. LEB 2221. Foncebadón; 21-VII-1978. F. Llamas. LEB 15197. Villacontilde-Valle de Mansilla (30TUN0512); 25 VI-1992. Encinar. E. de Paz. LEB 56056.

Marsilea strigosa Willd.

LEÓN: Laguna Amor. Valdemora (30TTM97); 
10-VIII-1988. Laguna temporal. A. Penas, M.E. García \& L. Herrero. LEB 44118. Laguna Amor. Valdemora (30TTM97); 11-IX-1985. IsoetoNanojuncetea. A. Penas, M.E. García, L. Herrero \& M. Garzón. LEB 38887. Manantial del Toro. Castilfalé (30TUM030737); 27-V-1996. Laguna. M.E. García \& E. de Paz. LEB 63260. Navianos de la Vega (30TTM67); 14-V-1985. A. Penas, M.E. García, L. Herrero, M. Garzón \& I. Jiménez. LEB 16016. Valdemora (30TTM97); 8-VIII-1978. Pastizales terofíticos inundados periódicamente. A. Penas. LEB 4835.

\section{* Mirabilis jalapa L.}

LEÓN: Vilecha; 19-VIII-1984. Junto a edificación del pueblo. F. Gómiz. LEB 26907.

\section{Monotropa hypopitys L.}

LEÓN: Almanza (30TUN32); 16-VI-1988. F. Llamas \& C. Acedo. LEB 46492. Campo el Arca. Cistierna (30TUN2742); 17-VII-1994. Pinar de repoblación. $1300 \mathrm{~m}$. R. Alonso. LEB 63452. Gambrillas. Villimer (30TUN039180); 12-VI-1994. Quejigar. E. de Paz. LEB 58925.La Candamia; 24VI-1984. Pinar. F. Gómiz. LEB 26918. Las Salas (30TUN25); 25-VII-1981. F.J. Pérez Carro. LEB 14393.

\section{Monotropa hypopitys L. var. hypopitys}

LEÓN: Morgovejo (30TUN3847); 12-VIII1997. Pinar de repoblación. $1200 \mathrm{~m}$. R. Alonso. LEB 63472.

\section{Myosurus minimus L.}

LEÓN: Carbajal de Fuentes; 20-VII-1978. Zonas encharcadas y luego desecadas. A. Penas. LEB 5941. Monte de la Vizana (30TTM66); 14-V1985. A. Penas, M.E. García, M. Garzón \& I. Jiménez. LEB 46698. Navianos de la Vega (30TTM67); 14-V-1985. A. Penas, M.E. García, M. Garzón \& I. Jiménez. LEB 46692. Santa Colomba de Somoza; 7-VI-1975. F. Llamas. LEB 607. Santa Colomba de Somoza; 7-VI-1975. F. Llamas. LEB 8970. Soto y Amío (30TTN64); 7-VI-1986. T.E. Díaz et al. LEB 32897. Val de San Lorenzo; 20-V1969. J. Andrés. LEB 6166. Valcavado del Páramo; 11-V-1972. J. Andrés. LEB 22199. Valcavado del Páramo; 11-V-1972. J. Andrés. LEB 23015. Valdepolo (30TUN11); 28-V-1985. Laguna. A. Penas. LEB 43335.

\section{Myriophyllum alterniflorum DC.}

LEÓN: Chana de Somoza; 21-VII-1982. F. Llamas. LEB 42985. Chozas de Arriba (30TTN772116); 19-VII-1981. Laguna. 890 m. M. Fernández Aláez. LEB 28164. Destriana; 11-VIII1977. F. Llamas. LEB 8241. Destriana; 14-VII-1977. F. Llamas. LEB 8739. Lago de Isoba (30TUN116687); 18-VI-1981. Interior del lago. M. \& C. Fernández Aláez. LEB 48570. Laguna de la Balastrera. Santas Martas (30TUN025038); 14-VII-1982. Interior de la laguna. M. \& C. Fernández Aláez. LEB 54458. Laguna de Valdearcos (30TUN00); 19-VI-1982.810 m. M. \& C. Fernández Aláez. LEB 30148. Laguna de Villadangos (30TTN721114); 8-V-1982. Interior de la laguna. M. Fernández Aláez. LEB 45387. Laguna del Redos. Villamuñío (30TUN176052); 30-VI-1982. Interior de la laguna. $M . \&$ \& . Fernández. Aláez. LEB 48777. Laguna El Estrorrubio. El Burgo Ranero (30TUN 10); 24-VI-1982. 900 m. M. \& C. Fernández Aláez. LEB 28351. Laguna Santiz. Valdepolo (30TUN11); 7-VII-1982. Interior de la laguna. $940 \mathrm{~m}$. M. Fernández Aláez. LEB 28351. Santa Cristina del Páramo (30TTM78); 20-VI-1982. Interior de la laguna. M. Fernández Aláez. LEB 35627. Villaverde la Chiquita (30TUN21); 10-VII-1982. Interior de la laguna. $M$. Fernández Aláez. LEB 35555.

\section{* Myriophyllum verticillatum $\mathrm{L}$.}

LEÓN: Lagunas de Palanquinos; 2-X-1983. Comunidades de la clase Potametea. J. Pérez Carro \& M.P. Fernández Areces. LEB 17923. LEB 17924. Citas bibliográficas: Pérez Carro et al. (1985)

\section{* Nigella gallica L.}

LEÓN: Puente Castro (30TTN91); 13-VII1977. Cultivos cerealistas sobre sustratos margosos. A. Penas. LEB 9013.

Citas bibliográficas: Penas Merino (1984).

\section{* Nymphaea alba L.}

LEÓN: Lago Somido. Las Médulas; 2-VI1981. F. Llamas. LEB 42962. LEB 13352.

\section{* Oenothera glazioviana Micheli}

LEÓN: Villanueva del Arbol (30TTM92); 29 VI-1990. Borde de carretera. F. Llamas \& C. Acedo. LEB 46532.

\section{Ortegia hispanica $\mathrm{L}$.}

LEÓN: Ribaseca (30TTN81); 28-VI-1986. J. 
Andrés \& F. Llamas. LEB 33071. Secarejo (30TTN62); 10-VI-1986. Comunidades nitrófilas. A. Penas, M.J. López Pacheco \& A. Terrón. LEB 32833. Velilla de la Reina (30TTN71); 10-VI-1986. J. Andrés \& F. Llamas. LEB 33070. Villadangos (30TTN71); 10-VI-1986. Comunidad nitrófila. E. Puente, C. Pérez \& L. Herrero. LEB 32834.

\section{* Paronychia rouyana Coincy}

LEÓN: Felechares de la Valdería (29TQG47); 23-VI-1987. Isoeto. 860 m. A. Penas, M.E. García \& L. Herrero. LEB 39264. Sub P. echinulata. Monte del Duque. Valderas (30TTM96); 22-VI-1988. Claro de encinar. A. Penas, M.E. García \& L. Herrero. LEB 44565. Sub $P$. echinulata.

\section{* Pinus pinea $\mathrm{L}$.}

LEÓN: Lago de Carucedo (29TQH80); 18-IX1983. Pinar. M.E. García. LEB 20110.

Citas bibliográficas: García González (1988)

Platycapnos spicata (L.) Bernh.

LEÓN: Monte del Duque. Valderas (3TTM9566); 12-IV-1995. Pradera en claro de encinar con ligera humedad. E. de Paz. LEB 59433. Monte Grande. Valencia de Don Juan (30TTM9479); 4-VI-1995. Campo arado, sobre margas. E. de Paz. LEB 59464. Monte Grande. Valencia de Don Juan; 13-V-1978. A. Penas. LEB 5968.

\section{Polycarpon tetraphyllum (L.) L. subsp. tetraphyllum}

LEÓN: Castropodame; 31-V-1986. Borde de camino. A. Penas, M.E. García \& L. Herrero. LEB 32099. León (30TTN81). 14-V-1988. A. Penas. LEB 44582 .

\section{Polygonum hydropiper $\mathrm{L}$.}

LEÓN: Destriana; 3-IX-1979.F. Llamas. LEB 13445. Valdefuentes del Páramo (30TTM68); 4-IX1985. Bidentetea tripartiti. 790 m. M.E. García. LEB 40939.

\section{Populus alba $\mathrm{L}$.}

LEÓN: Carbajal de la Legua (30TTN82); 14VIII-1985. Populion albae. 870 m. M.E. García. LEB 40406. Castrofuerte (30TTM97); 20-IV-1985. J. Andrés. LEB 46339. Cistierna (30TUN2640); 21 VI-1994. Borde de carretera. $980 \mathrm{~m}$. R. Alonso. LEB 63454. La Vecilla de Curueño (30TUN04); 6-
VI-1982. Borde de río. M.J. López Pacheco. LEB 16232. Pombriego (29TPG89); 12-VII-1986. Olmeda. I. Jiménez. LEB 32659. Valencia de Don Juan; 1-V-1985. T.E. Díaz \& col. LEB 37477.

\section{* Portulaca oleracea L.}

LEÓN: Destriana; 11-VIII-1977. F. Llamas. LEB 9049. Fresno de la Vega; 28-VIII-1979. A. Penas. LEB 5883. Palanquinos (30TTN90); 9-X1984. A. Penas, L. Herrero, M. Garzón \& I. Jiménez. LEB 46857.

Citas bibliográficas: Llamas (1984).

Ranunculus abnormis Cutanda \& Willk.

LEÓN: Santa Colomba de Somoza; 15-V-1974. $J$. Andrés et al. LEB 15337. LEB 15338. LEB 10594. Santa Colomba de Somoza; 1-V-1975. Prado de diente. F. Llamas. LEB 718. LEB 15163.

Ranunculus ollisiponensis Pers. subsp. alpinus (Boiss. \& Reuter) Grau

LEÓn: Rosales; 14-VI-1974. A. Pérez. LEB 795. Villaobispo de las Regueras (30TTN929217); 3-IV-1993. Aulagar. E. de Paz. LEB 55663.

\section{Reseda phyteuma $\mathrm{L}$.}

LEÓN: Colinas de Lancia (30TUN01); 16-V1978. J. Andrés. LEB 41606. Colinas de Lancia; $17-$ IV-1983. Sustratos margosos. A. Penas, R. García Cachán \& M.E. García. LEB 15461. Lancia (30TUN003112); 22-V-1993. Pastizal. E. de Paz. LEB 56000. Mansilla de las Mulas (30TUN00); 22 IV-1977. A. Penas. LEB 38882. Villafalé (30TUN01); 1-V-1986. Comunidades nitrófilas. A. Penas, M.J. López Pacheco \& A. Terrón. LEB 32161. Villafañe (30TUN01); 12-V-1987. Matorral de Genista scorpius. J. Andrés. LEB 44545.

\section{Rorippa amphibia (L.) Besser}

LEÓN: Bracas (30TTM98); 24-VIII-1977. En los márgenes del río Esla. A. Penas. LEB 4845. Bracas (30TTM98); 24-VIII-1977. Medio acuático con cierto índice de subsalinidad. A. Penas. LEB 34554. Palanquinos (30TTN90); 24-VIII-1977. En el río. A. Penas. LEB 41059.

\section{* Rorippa microphylla (Boenn. ex Rchb.) Hyl.}

LEÓN: Gradefes (30TUN12); 29-V-1990. Pedregal de río. F. Llamas, C. Acedo \& G. González Sierra. LEB 54832. 


\section{Sagina procumbens $\mathrm{L}$.}

LEÓN: Abelgas (30TTN55); 13-VIII-1989. Talud rezumante. E. Puente \& M.J. López Pacheco. LEB 48453. Barniedo de la Reina-Portilla de la Reina (30TUN4764); 31-VII-1996. Paredón rezumante silíceo. $1215 \mathrm{~m}$. R. Alonso. LEB 63469. Chozas de Arriba (30TTN7711); 4-VII-1981. Pradera que bordea la laguna. M. Fernández Aláez. LEB 35679. Chozas de Arriba (30TTN772113); 26VII-1981. Pradera que bordea la laguna. $M$. Fernández Aláez. LEB 36178. La Mata de Monteagudo (30TUN3344); 4-VIII-1997. Fuente. $1150 \mathrm{~m}$. R. Alonso. LEB 63475. Laguna de Chozas de Arriba (30TTN772116); 19-VII-1981. Pradera de borde de laguna. 890 m. M. Fernández Aláez. LEB 28179. Palacios del Sil (29TQH05); 2-VII1983. Muro de fuente. E. Puente. LEB 16656. Puerto de Leitariegos (29TQH16); 17-VI-1982. Zonas pisoteadas. E. Puente. LEB 13812. Puerto de Vegarada; 2-VII-1973. La Blanca. LEB 25653. Rosales; 14-VI-1974. C. Pérez Morales. LEB 25655. Valle de Fontaninas. Tejedo del Sil (29TQH15); 2 VII-1983. Lugar húmedo. E. Puente. LEB 16843. Valle de Valponguero. Barniedo de la Reina (30TUN5362); 16-VIII-1997. Aguas nacientes. 1680 m. R. Alonso. LEB 63471. Villamanín (30TTN85); 16-VII-1983. Intersticios de empedrados. C. Pérez. Morales. LEB 25404.

\section{* Salsola kali L.}

LEÓN: Castrofuerte (30TTM97); 1 1-IX-1985. Ruderali-Secalietea. 750 m. M.E. García. LEB 36234. Fresno de la Vega (30TTM99); 23-VIII1979. En comunidades nitrófilas salobres como mala hierba de cultivo hortícola sobre arenas de origen fluvial. A. Penas. LEB 9012. Fresno de la Vega; $28-$ VIII-1979. F. Llamas. LEB 25549.

Citas bibliográficas: Penas Merino (1984).

\section{Samolus valerandi L.}

LEÓN: Laguna Grande. Bercianos del Real Camino (30TUM29); 5-VII-1981. Borde de la laguna y zona de transición. $830 \mathrm{~m}$. M. Fernández Aláez. LEB 28328.

\section{Saxifraga longifolia Lapeyr.}

LEÓN: La Cueta; 15-VIII-1984. Altas rocas calizas. C. Romero. LEB 1384. La Cueta (29TQH36); 9-VIII-1984. Calizas. 2000 m. E. Puente. LEB 26263.
Saxifraga praetermissa D.A. Webb

LEÓN: Pedavejo. Vega de Liordes; 30-VII1981. F. Llamas. LEB 37606. Peña Remoña; $28-$ VII-1981. M.T. Ponga. LEB 23767. Peñasanta; $18-$ VII-1980. Roca caliza. Medio húmedo. J. Andrés \& A. González Ordás. LEB 23038. Pico Huevo (30TTN96); 19-VII-1979. M.J. López Pacheco. LEB 17431. Puerto de Vegarada; I-VIII-1981. M.T. Ponga. LEB 23775. Torre Peñalba; 27-VIII-1981. M.T. Ponga. LEB 23766.

\section{Sedum aetnense Tineo}

LEÓN: Morales del Arcediano; 26-III-1982. T.E. Díaz \& F. Llamas. LEB 8363. Morales del Arcediano; 26-III-1982. F. Llamas. LEB 15183. Morales del Arcediano (29TQG3999); 21-III-1977. F. Llamas. LEB 8362.

\section{Sedum caespitosum (Cav.) DC.}

LEÓN: Colinas de Lancia; 9-V-1983. T.E. Díaz \& al.. LEB 18000. El Otero de Valdetuéjar (30TUN3444); 25-V-1996. Pastizal anual acidófilo. 1030 m. R. Alonso. LEB 63446. Quintanilla de Los Oteros (30TTM9986); 9-V-1998. Pastizal terofítico basófilo. R. Alonso, E. Alonso \& E. Puente. LEB 63261. Vegas del Condado; 19-IV-1970. J. Andrés \& R. Carbó. LEB 14512. Viloria de la Jurisdicción (30TTN80); 19-IV-1985. A. Penas et al. LEB 42062.

\section{* Sedum maireanum Sennen \\ LEÓN: Santa Colomba de Somoza; 7-VI-1975. F. Llamas. LEB 14514. Sub. S. lagascae. Villadangos del Páramo (30TTN71); 22-V-1985. A. Penas, M.E. García, L. Herrero, M. Garzón \& I. Jiménez. LEB 29244. Sub. S. lagascae.}

\section{Silene conica L. subsp. conica}

LEÓN: Ardón; 6-VI-1978. A. Penas. LEB 5901. Cistierna; 30-V-1982. J. Andrés. LEB 13965. La Virgen del Camino; 12-V-1973. J. Andrés \& $R$. Carbó. LEB 471. León-Asturias. Km. 3 (30TUN880230); 27-V-1998. Herbazal. E. de Paz \& P. Sastre. LEB 63259.

\section{Silene conoidea $\mathrm{L}$.}

LEÓN: El Chopo. Villasabariego (30TUN028131); 19-VI-1992. Encinar. E. de Paz. LEB 55682. Matallana de Valmadrigal; 2-VI-1983. Campo de cultivo. T.E. Díaz, A. Penas, E. Puente \& M.E. García. LEB 26873. Santas Martas; 7-VI- 
1977. Borde de sembrado. A. Penas. LEB 5900. Villacontilde (30TUN01); 16-V-1989. Campo de cultivo ceralista sobre margas miocénicas. A. Penas \& M.E. García. LEB 45215.

\section{Silene mellifera Boiss. \& Reuter}

LEÓN: Vegacervera; 11-VII-1970. J. Andrés \& R. Carbó. LEB 483. Monte Grande. Valencia de Don Juan; 3-VI-1976. A. Penas. LEB 11832. Sub S. italica. Monte Grande. Valencia de Don Juan (30TTM97). 25V-1977. A. Penas. LEB 46065. Sub S. italica.

\section{Silene muscipula L.}

LEÓN: Monte Grande. Valencia de Don Juan; 25-V-1977. En claros de encinar. A. Penas. LEB 4848.

\section{Sisymbrium orientale $\mathrm{L}$}

LEÓN: Gordaliza del Pino (30TUN29); 16III-1990. Viñas abandonadas. F. Llamas \& C. Acedo. LEB 32672. Vega de Gordón (30TTN85); 12-VI1983. Ruderal. C. Pérez Morales. LEB 25144. Zalamillas (30TUM08); 21-V-1985. T.E. Díaz, M.E. García \& R. García. LEB 32061.

\section{Thlaspi alliaceum $\mathrm{L}$.}

LEÓN: Las Llanas (30TUN14); 2-V-1987. Borde de camino. L. Herrero. LEB 35205. Llama de Coqueros; 5-VI-1983. T.E. Díaz, A. Penas \& M.E. García. LEB 21813. Llama de Coqueros; 5-VI1983. T.E. Díaz, A. Penas \& M.E. García. LEB 21814. Riaño; 21-VI-1986. Comunidad nitrófila. $E$. Puente, C. Pérez \& L. Herrero. LEB 32765.

\section{Ulmus minor Mill.}

LEÓN: Castrillo de Cabrera; 18-IV-1992. Olmeda. 950 m. A. Penas et al. LEB 48309. Cerezal (30TUN3638); 6-IX-1997. Sebe. 990 m. R. Alonso. LEB 63456. El Otero de Valdetuéjar (30TUN3444); 7-VIII-1997. Borde de prado. 1015 m. R. Alonso. LEB 63473. La Mata de Monteagudo (30TUN3245); 12-IV-1994. Borde de prado. $1100 \mathrm{~m}$. R. Alonso. LEB 63455. La Valcueva (30TTN94); 5-X-1982. Borde de camino. M.J. López Pacheco. LEB 17393. León. II-IV-VI-1985. En cuneta de carretera. $F$. Gómiz. LEB 30218. Mirantes de Luna; 18-VIII1973. Lugares frescos. C. Romero. LEB 130. Mirantes de Luna; 18-VIII-1983. Bosques frescos. C. Romero. LEB 18929. Morgovejo (30TUN3945); 14-X-1994. Borde de muro. $1040 \mathrm{~m}$. R. Alonso. LEB 63453. Pedredo; 29-III-1976. F. Llamas. LEB
8723. Pombriego (29TPG89); 12-VII-1986. Olmeda. I. Jiménez. LEB 32657. Quintana de la Peña (30TUN3039); 5-VIII-1995. Chopera. $1170 \mathrm{~m}$. $R$. Alonso. LEB 63442. Robles de Laciana (29TQH25); 17-VII-1984. Fresneda. E. Puente. LEB 24245. Rucayo; 3-VII-1975. E. Hernández. LEB 27223. Sancedo (29TPH92); 5-VIII-1985. Olmeda. 675 m. M.E. García LEB 49448. Sancedo (29TPH92); 5VIII-1985. Querco-Fagetea. 675 m. M.E. García. LEB 37155. Tonín (30TTN86); 4-VIII-1977. Bosque ribereño. C. Pérez Morales. LEB 27373. Valencia de Don Juan; 2-V-1971. J. Andrés \& R. Carbó. LEB 37710. Valencia de Don Juan-Villamañán (30TTM88); 20-IV-1985. T.E. Díaz. LEB 44688. Valmartino-Quintana de la Peña. Cruce (30TUN2938); 28-VIII-1996. Sauceda-chopera. $1040 \mathrm{~m}$. R. Alonso. LEB 63465. Villanueva de Carrizo (30TTN61); 28-V-1986. 900 m. M.E. García. LEB 50056. Villanueva de Carrizo (30TTN61); 28-V-1986. Querco-Fagetea. $900 \mathrm{~m}$. M.E. García. LEB 35759.

\section{Vaccaria hispanica (Miller) Rauschert}

LEÓN: Castilfalé (30TTM995778); 8-VI1994. Pastizal de laguna. E. de Paz. LEB 58899. Cifuentes de Rueda (30TUN11); 4-VI-1986. Nitrófila. J. Andrés, F. Llamas \& D. Fernández. LEB 32919. Monte Grande. Valencia de Don Juan (30TTM9479); 4-VI-1995. Sobre margas con cantos, arado. E. de Paz. LEB 59466. Monte Grande. Valencia de Don Juan; 3-VI-1976. A. Penas. LEB 38202. Monte Grande. Valencia de Don Juan; 7-VI1977. Sembrado. A. Penas. LEB 5898. Santas Martas (30TUN00); 30-VI-1983. Talud de carretera. $A$. Penas \& M.E. García. LEB 41961. Santas Martas (30TUN00); 30-VI-1983. Talud de carretera. A. Penas \& M.E. García. LEB 26855. Valdelafuente; 30-VI-1974. J. Andrés. LEB 10592.

Xolantha globulariifolia (Lam.) Gallego

LEÓN: Foncebadón (29TQH10); 17-X-1982.

Brezal. M.E. García. LEB 19524.

\section{BIBLIOGRAFÍA}

AEDO, C., J.J. ALDASORO, J.M. ARGÜELLES, J.L. DÍAZ ALONSO, A. DÍEZ RIOL, J.M. GONZÁLEZ DEL VALLE, M. LAÍNZ, G. MORENO MORAL, J. PATALLO y O. 
SÁNCHEZ PEDRAJA -1994- Contribuciones al conocimiento de la flora cantábrica, II. Fontqueria 40: 67-100. Madrid.

AEDO, C., J.J. ALDASORO, J.M. ARGÜELLES, J.L. DÍAZ ALONSO, A. DÍEZ RIOL, J.M GONZÁLEZ DEL VALLE, M. LAÍNZ, G. MORENO MORAL, J. PATALLO y O. SÁNCHEZ PEDRAJA -1997- Contribuciones al conocimiento de la flora cantábrica, III. Anales Jard. Bot. Madrid 55 (2): 321-350.

CARBÓ NADAL, R., M. MAYOR LÓPEZ, J. ANDRÉS RODRÍGUEZ y J.M. LOSA QUINTANA -1977- Aportaciones al catálogo florístico de la provincia de León. II. Acta Bot. Malacitana 3: 63-120. Málaga.

CARBÓ, R. y M. MAYOR, J. ANDRÉS y J.M. LOSA - 1972- Aportaciones al catálogo florístico de la provincia de León. Anal. Fac. Veter. León 18 (1): 225-352.

CASTROVIEJO. S., M. LAÍNZ, G. LÓPEZ GONZÁLEZ, P. MONTSERRAT, F. MUÑOZ GARMENDIA, J. PAIVA y L. VILLAR (eds.) 1986, 1990, 1993, 1997-Flora iberica. Plantas vasculares de la Península Ibérica e Islas Baleares. Vol. I, II, III, IV, V y VIII. Madrid. DÍAZ GONZÁLEZ, T.E. y A. PENAS MERINO 1984- Datos sobre la vegetación terofítica y nitrófila leonesa. Acta Bot. Malacitana 9: 233 254. Málaga.

FERNÁNDEZ ARECES, M.P. -1989- Flora y Vegetación rupícola de la Cordillera Cantábrica, Montes de León y cuenca alta del río Ebro. Revisión taxonómica del género Saxifraga L. sección Dactyloides Tausch en el norte de la Península Ibérica. Memoria Doctoral publicada en microficha. Universidad de León.

FERNÁNDEZ CASAS, J., R. GAMARRA y $\mathrm{M}^{\mathrm{a}} \mathrm{J}$. MORALES ABAD, eds. -1994- Asientos para un atlas corológico de la flora occidental, 21. Fontqueria 39: 281-394. Madrid.

GARCÍA GONZÁLEZ, M.E. -1988-Efectos de las repoblaciones con pinos en la clímax de la Quercetea ilicis mediterránea leonesa. Excma. Diputación Provincial de León. Inst. Fray Bernardino de Sahagún.

GARCÍA GONZÁLEZ, M.E., E. PUENTE GARCÍA, M.J. LÓPEZ PACHECO, L. HERRERO CEMBRANOS y A. PENAS MERINO -1987De Plantis Legionensibus. Notula X: Studia Botanica 6: 103-108. Salamanca.
HERNÁNDEZ HERNÁNDEZ, E. - 1985- De Plantis Legionensibus. Nota IV. Acta Bot. Malcitana 10: 45-48. Málaga.

HERRERO, L., M.E. GARCÍA GONZÁLEZ y A. PENAS -1988- De Plantis Legionensibus. Notula XI. Acta Bot. Malacitana 13: 319-323. Málaga.

LLAMAS, F. -1984- Flora y Vegetación de la Maragatería; Excma. Diputación Provincial de León. Inst. Fray Bernardino de Sahagún.

LLAMAS, F., R. CARBÓ y J. ANDRÉS -1984Contribución al conocimiento de la flora de León (España). Lazaroa 6: 283-285. Madrid.

PENAS MERINO, A. -1984- Nuevos táxones para la flora leonesa. Lagascalia 13 (1): 3-16. Sevilla.

PENAS MERINO, A., M.E. GARCÍA GONZÁLEZ, L. HERRERO CEMBRANOS, M. GARZÓN DE PAZ y I. JIMÉNEZ VICENTE - 1987, 1988- De Plantis Legionensibus. Notula IX. Lazaroa 10: 299-302. Madrid.

PÉREZ CARRO, F.J., M.P. FERNÁNDEZ ARECES y T.E. DÍAZ GONZÁLEZ - 1985- De Plantis Legionensis. Notula II. Studia Botanica 4: 137 142. Salamanca.

PÉREZ MORALES, C. -1988- Flora y Vegetación de la cuenca alta del río Bernesga. Dip. Prov. León. Inst. Fray Bernardino de Sahagún.

PÉREZ MORALES, C., L. HERRERO CEMBRANOS, M.E. GARCÍA GONZÁLEZ, E. PUENTE GARCÍA y A PENAS MERINO 1993- De Plantis Legionensibus. Notula XIV. Botanica Complutensis 18: 259-262. Madrid.

SILVA PANDO, F.J. -1994- Flora y series de vegetación de la Sierra de Ancares. Fontqueria 40: 233-388.

TÜXEN, R. \& E. OBERDORFER - 1958- Eurosibirische Phanerogamen-Gesellschaften Spaniens. Veröff. Geobot. Inst. Rübel Zürich 32: 1-328.

Aceptado para su publicación en Septiembre de 1998

Dirección de los autores. Departamento de Biología Vegetal (Botánica). Facultad de Biología. Universidad de León. Campus de Vegazana $s / n$. 24071-León (España). 